\title{
Chemo-mechanical model for skeletal muscle contraction
}

\author{
M. Karami ${ }^{1,2}$, B. Calvo ${ }^{1}$, H. Zohoor ${ }^{2}$, K. Firoozbakhsh ${ }^{2}$, J. Grasa ${ }^{1}$ \\ ${ }^{1}$ Applied Mechanics and Bioengineering (AMB) \\ Instituto de Investigación en Ingeniería de Aragón (I3A) \\ Universidad de Zaragoza, Mariano Esquillor s/n, 50018, Zaragoza, Spain. \\ Tel. +34-976762707, e-mail: m_karami@unizar.es \\ ${ }^{2}$ School of Mechanical Engineering, Sharif University of Technology, Tehran, Iran
}

\section{Abstract}

Generation of force in skeletal muscle tissue depends on both chemical and mechanical phenomena. In this work, considering the kinetics of cross bridges, a one dimensional model has been proposed for predicting the isometric force according to the intracellular calcium ion distribution.

\section{Introduction}

The different ways of skeletal muscle activation lead to the formation of cross bridges (XB) according to the available calcium ion flow. XB kinetics is the cyclic connection between thin and thick filament at the sarcomere level to produce mechanical force.

Since the Huxley model (Huxley 1974) can be considered as the first numerical muscle model considering $\mathrm{XB}$ kinetics, several works have developed different XB formulations (Razumova et al. 1999; Shorten et al. 2007). Recently, a more realistic 3D model (Heidlauf and Röhrle 2014) have appeared providing a chemo-mechanical approach to simulate complex muscle geometries. In this work, a new thermodynamically consistent model is proposed incorporating different scales to reproduce the force developed by muscle tissue.

\section{Material and Methods}

\section{Cross bridge (XB) kinetics}

Mechanical force in skeletal muscle is produced during the well known power stroke, which is the transition step between chemical and mechanical energy. Consequently, a chemical model should be able to consider this event. Therefore, XB kinetics model of Razumova et al. 1999 (Fig. 1) has been used as the base of the chemical part. This model considers four states, including the pre- and post- power stroke. The first state $\left(\mathrm{R}_{\text {off }}\right)$ corresponds to the fraction of thin-filament regulatory units (RU) which are blocked by tropomyosin-troponin complex (TTC) (off position). In the second state (D) $\mathrm{Ca}^{+2}$ has bound to TTC, RU changes to on position but there is no attachment. $A_{1}$ and $A_{2}$ states include cross bridge formation. Attached $\mathrm{XB}$ can be in two situations, pre- and post-power stroke. The fractions of these situations are defined respectively with $A_{1}$ and $A_{2}$.

These states are in a cyclic dynamics and different rates (Fig. 1) control the number of XBs in the four situations. $K_{\text {on }}$ and $\mathrm{k}_{\text {off }}$ rates manage the on and off condition of RUs according to the calcium concentration level.

The concentration of XBs in the four different situations can be found by solving a system of three ordinary equations as:

$$
\begin{aligned}
& \dot{D}(t)=k_{\text {on }} R_{\text {off }}(t)+f^{\prime} A_{1}(t)+g A_{2}(t)-\left(k_{\text {off }}+f\right) D(t) \\
& \dot{A}_{1}(t)=f D(t)+h^{\prime} A_{2}(t)-\left(f^{\prime}+h\right) A_{1}(t) \\
& \dot{A}_{2}(t)=h A_{1}(t)-\left(h^{\prime}+g\right) A_{2}(t)
\end{aligned}
$$$$
\text { and considering the constraint } 1=\mathrm{R}_{\mathrm{off}}+\mathrm{D}+\mathrm{A}_{1}+\mathrm{A}_{2} \text {. }
$$

\section{Mechanical Model}

Deriveing from the first and second law of thermodynamic, we have obtained the following constitutive relations:

$$
\begin{aligned}
& P=\frac{\partial}{\partial \lambda}\left(\psi_{p}(\lambda)+N\left(\lambda_{a}\right) \psi_{a}\left(\lambda_{e}, S_{i}\right)\right) \\
& P_{a}-\frac{\partial}{\partial \lambda}\left(N\left(\lambda_{a}\right) \psi_{a}\left(\lambda_{e}, S_{i}\right)\right)=C \dot{\lambda}_{a}
\end{aligned}
$$

where $\mathrm{S}_{\mathrm{i}}=\left(\mathrm{R}_{\text {off }}, \mathrm{D}, \mathrm{A}_{1}, \mathrm{~A}_{2}\right), \mathrm{P}$ and $\mathrm{P}_{\mathrm{a}}$ are total and active force; $\mathrm{N}$ is the force-length relationship in skeletal muscle and

$\psi_{p}=\left(e^{(\lambda-1)}-\lambda\right)$ 
$\psi_{a}=E\left(A_{1}+A_{2}\right) \frac{1}{2}\left(\lambda_{e}-e\right)^{2}$

$P_{a}=-v A_{2} A_{1} / \max \left(A_{1}\right)$

$C=F\left(A_{1}+A_{2}\right) N\left(\lambda_{a}\right) \geq 0$

$\mathrm{E}, \mathrm{F}$ and $v$ are three constants of the model.

Model parameters, including those for the calcium distribution which is involved in the transition from $\mathrm{R}_{\text {off }}$ state to D are listed in Table 1.

\section{Results}

The simulated stress was compared with the experimental data of Extensor digitorum longus (EDL) muscle under an isometric contraction (Sierra et al. 2016) (Fig. 2). As can be observed the result is in good agreement with the real muscle behavior.

\section{Discussion}

The model can predict accurately the isometric stress (Fig. 2). However, one important limitation is that no experimental based calcium distribution has been considered in the model.

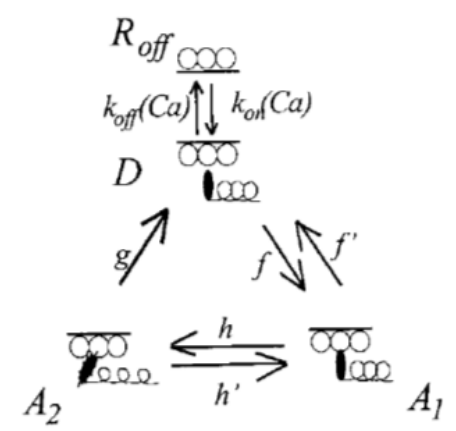

Figure 1 four state XB kinetics (Razumova et al. 1999)

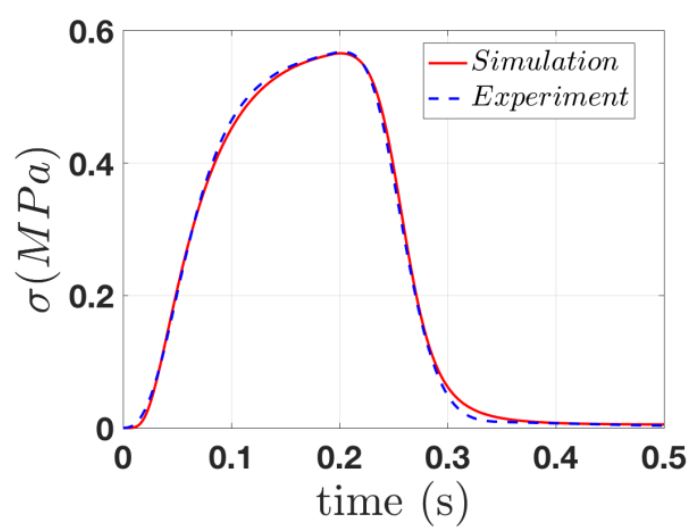

Furthermore, if the stimulation mechanism is added, the model will be able to consider what happens before the force generation and therefore all the contraction stages. The model in the current form can be used to study calcium related phenomena such as fatigue.

\section{References}

HEIDLAUF, T. AND O. RÖHRLE A multiscale chemoelectro-mechanical skeletal muscle model to analyze muscle contraction and force generation for different muscle fiber arrangements. Frontiers in Physiology, 2014, 5, 498.

HUXLEY, A. Muscular contraction. The Journal of physiology, 1974, 243(1), 1.

RAZUMOVA, M. V., A. E. BUKATINA AND K. B. CAMPBELL Stiffness-distortion sarcomere model for muscle simulation. Journal of Applied Physiology, 1999, 87(5), 18611876.

SHORTEN, P. R., P. O'CALLAGHAN, J. B. DAVIDSON AND T. K. SOBOLEVA A mathematical model of fatigue in skeletal muscle force contraction. Journal of muscle research and cell motility, 2007, 28(6), 293-313.

SIERRA, M., J. GRASA, M. MUÑOZ, F. MIANA-MENA, et al. Predicting muscle fatigue: a response surface approximation based on proper generalized decomposition technique. Biomechanics and modeling in mechanobiology, 2016, 1-10.

Table 1. arameter of the model

\begin{tabular}{|c|c|c|c|}
\hline Parameter & Value & Parameter & Value \\
\hline $\mathrm{Ca}_{\text {th }}(\mu \mathrm{M})$ & 0.575 & $k_{\text {off }}^{C a}(1 / s)$ & 150 \\
\hline $\mathrm{Ca}_{50}(\mu \mathrm{M})$ & 1.48 & $k_{\text {off }}^{0}(1 / s)$ & 450 \\
\hline $\mathrm{Ca}_{\max }(\mu \mathrm{M})$ & 10 & $k_{\text {on }}^{C a}(1 / s)$ & 450 \\
\hline $\mathrm{t}_{\mathrm{ca} \text { max }}(\mathrm{s})$ & 0.195 & $k_{\text {on }}^{0}(1 / \mathrm{s})$ & 0 \\
\hline $\mathrm{a}$ & 0.304 & $\mathrm{f}(1 / \mathrm{s})$ & 150 \\
\hline $\mathrm{b}$ & 38 & $\mathrm{f}^{\prime}(1 / \mathrm{s})$ & 1500 \\
\hline $\mathrm{E}$ & 400 & $\mathrm{~g}(1 / \mathrm{s})$ & 12 \\
\hline $\mathrm{F}$ & 1 & $\mathrm{~h}(1 / \mathrm{s})$ & 24 \\
\hline$v$ & 9.3 & $\mathrm{~h}^{\prime}(1 / \mathrm{s})$ & 18 \\
\hline
\end{tabular}

Figure 2 comparssion between experimental (dashed line) and simulated (solid line) stress of isometric contraction in EDL of rabbit 